\title{
Effect of earth grid conductor spacing on the safety criteria of substation earthing
}

\begin{abstract}
Site dependent parameters such as soil resistivity, maximum grid current, fault duration, shock duration, surface layer material resistivity, and grid geometry have substantial impact on the design and performance of an earth grid. The grid geometry, i.e. the area occupied by the grid system, conductor spacing and the depth of burial of earth grid specifically have much impact on the mesh, step, touch voltage and earth potential rise, while parameters such as the conductor diameter and the thickness of the surfacing material have less impact on the safety criteria. In this paper, a distribution substation earth grid was designed using the CDEGS software by varying the spacing between grid conductors (compression ratio) from 1 to 0.5 in steps of 0.1 . The safety criteria were studied under three scenarios, without surface layer material, with surface layer material of resistivity $3000 \mathrm{q}-\mathrm{m}$, and surface layer material of resistivity 5000q-m. Results indicated that the grid resistance and earth potential rise were lower for a compression ratio 0.9 , while the step and touch voltages were found to be similar for each case of surface layer material.
\end{abstract}

Keyword: Conductor spacing; Safety criteria; Earth grid; Substation earthing 